\title{
Trayectorias personalizadas docentes: fortalecimiento flexible en la práctica docente
}

\author{
María Concepción Barrón Tirado y Francisco Cervantes Pérez
}

\begin{abstract}
Resumen
En este artículo se presenta, de manera general, el Programa Estratégico de Apoyo a la Docencia (PEAD): "Docencia y aprendizaje en la era digital: trayectorias docentes personalizadas", como una propuesta integral y flexible para el fortalecimiento de la práctica docente bajo los principios de equidad, inclusión y atención a la diversidad. Se parte de considerar a los docentes como constructores de un saber disciplinario-didáctico en función de sus contextos de trabajo y como agentes de cambio continuo, por lo que un programa de esta naturaleza está orientado a fortalecer su desarrollo personal, social y profesional, con una visión ética, crítica y comprometida con la formación del ciudadano del mundo contemporáneo.

La principal aportación de esta propuesta reside en la articulación entre una visión de la formación docente desde la multirreferencialidad, una organización curricular flexible por ejes y por competencias, un aprendizaje a través de desafíos y actividades, así como un proceso de evaluación continuo; todo ello en el marco de una estructuración didáctica con base en cursos autogestivos y apoyada con el uso apropiado de las tecnologías digitales.
\end{abstract}

Palabras clave: trayectorias docentes personalizadas, formación docente, competencias docentes, innovación didáctica, autoorganización, autogestión, autorreferencia.

\section{Personalized teaChing trajeCtORIES: fleXIBle stRengthenING IN TEACHING PRACTICE}

\begin{abstract}
This article presents, in general, the Strategic Program for Teaching Support (PEAD, from its Spanish acronym): "Teaching and learning in the digital age: personalized teaching paths", as an integral and flexible proposal to reinforce teaching practice under the principles of equity, inclusion and attention to diversity. It is based on considering teachers as builders of disciplinary-didactic knowledge based on their work contexts, and as agents of continuous change. Therefore, a program of this nature aims to reinforce teachers' personal, social and professional development, with an ethical and critical vision committed with the formation of contemporary world citizens.

The main contribution of this proposal lies in the junction of a vision of teacher training from multireferentiality, a flexible curricular organization based on axes and competences, learning through challenges and activities, as well as a continuous evaluation process; all this within the framework of a didactic structuring based on self-managed courses and supported with the appropriate use of digital technologies.
\end{abstract}

Keywords: personalized teaching paths, teaching training, teaching competences, didactic innovation, self-organization, self-managed, self-reference.

Dol: http://doi.org/10.22201/codeic.16076079e.2019.v20n6.a3

Universidad Nacional Autónoma de México, Coordinación de Desarrollo Educativo e Innovación Curricular (CODEIC Este es un artículo de acceso abierto bajo la licencia de Creative Commons 4.0)(i)(2) 


\section{María Concepción Barrón Tirado}

http://orcid.org/0000-0003-4214-9228 barront@unam.mx

Secretaria Académica de la Coordinación de Universidad Abierta y Educación a Distancia e Investigadora del IISUE/UnAm. Doctora en Pedagogía por la Facultad de Filosofía y Letras de la unAm y SNI-II. Coordinadora académica del Proyecto "Docencia y aprendizaje en la era digital. Trayectorias docentes personalizadas" (CuAEd/UnAm). Profesora del Posgrado de Pedagogía de la unam. Sus líneas de investigación son el currículum, la didáctica y la formación profesional.

\section{Francisco Cervantes Pérez}

francisco cp@cuaed.unam.mx

Director General de la Coordinación de Universidad Abierta y Educación a Distancia de la unam. Doctorado en Ciencias de la Computación y de la Información por la Universidad de Massachusetts en Amherst, Mass., Estados Unidos, con la disertación: Modeling and analysis of neural networks in the visuomotor system of anuran amphibia. Profesor de Carrera Titular "B", Tiempo Completo, Escuela Nacional de Estudios Superiores, Unidad Morelia, UnAM.

\section{Introducción}

La sociedad del siglo xxi se encuentra inserta en un constante proceso de transformación, en gran medida debido al acelerado desarrollo de las Tecnologías de la Información y Comunicación (TIC) y las Tecnologías del Aprendizaje y el Conocimiento (TAC), lo que ha impactado las funciones sustantivas de instituciones educativas y los roles de docentes y alumnos, quienes han modificado sus prácticas cotidianas, así como sus ambientes de enseñanza y de aprendizaje ante la progresiva inclusión de las tecnologías en los procesos educativos.

Se ha afirmado que la intervención de las TIC Y TAc en las prácticas educativas tiene por objetivo preparar a los estudiantes para un nuevo tipo de sociedad la del aprendizaje y la información - no sólo a través de su uso directo, sino como herramientas que facilitan aprender de manera autónoma.

Considerando lo anterior, ¿cómo deberían prepararse los profesores?, ¿qué tipo de conocimientos, habilidades y competencias requieren para enfrentar los desafíos del presente siglo?, ¿a través de qué estrategias será posible adecuar tanto los conocimientos como las actitudes de los docentes con la finalidad de responder a los retos emanados de la sociedad del aprendizaje y de la información? Ante estas interrogantes, en la Coordinación de Universidad Abierta y Educación a Distancia (CUAED), de la Universidad Nacional Autónoma de México (UNAM), se ha diseñado una propuesta para fortalecer la práctica docente en el contexto de la sociedad del aprendizaje. 


\section{La incursión de las TIC y TAC en el ámbito educativo}

Es incuestionable que el avance de las tecnologías digitales ha modificado hasta las formas de trabajo desde el ámbito más cotidiano, lo que trae consigo una serie de cambios en las maneras de relacionarnos y, más aún, en la forma cómo aprendemos. Frente a este escenario, los fundamentos pedagógicos y educativos han sufrido cuestionamientos y modificaciones. Por un lado, existe incertidumbre sobre la capacidad de la escuela para renovarse ante la incursión de las tecnologías en el ámbito educativo y sobre su papel como institución promotora del desarrollo integral de las personas. Por otro, resulta innegable que han emergido propuestas educativas que modifican las perspectivas pedagógicas que tradicionalmente han regido las prácticas de enseñanza y aprendizaje, dando pauta a nuevas formas de pensar la educación, el currículum, las didácticas, y, por ende, los procesos de enseñanza, aprendizaje y evaluación.

Ante tal panorama, la universidad necesita transformarse de una "institución con énfasis en la enseñanza" a una "institución con énfasis en el aprendizaje", en un espacio donde todos sus miembros, no sólo los estudiantes y maestros, sino también los técnicos y los administradores, se mantengan unidos para aprender. Para ello, se requiere de la construcción de ambientes desde lo que César Coll (2013) ha denominado una nueva ecología del aprendizaje, que incorpora tres componentes fundamentales: a) el aprendizaje a lo largo y ancho de la vida, y la tendencia creciente a la informatización del aprendizaje; b) la construcción de trayectorias personales de aprendizaje como vías de acceso al conocimiento; y c) la adquisición y desarrollo de competencias genéricas y transversales relacionadas con la capacidad de aprender. Así,

Todos estos factores hacen que no haya dos trayectorias personales de aprendizaje idénticas, y lo que es aún más importante, que resulte problemático plantear y abordar el aprendizaje de las personas en uno de estos contextos — por ejemplo, en la escuela - sin tomar en consideración la trayectoria personal de conjunto en la que se inscribe (Coll, 2016, p. 34).

La incorporación de las TIC y las TAC en el ámbito educativo brinda la posibilidad de construir trayectorias personales de aprendizaje, pues el procesar en tiempo real una gran cantidad de datos, y transformarlos en información y conocimiento, permite a los participantes ajustar su proceso formativo con base en los avances obtenidos en cada momento.

\section{Docencia y aprendizaje en la era digital. Trayectorias docentes personalizadas}

La CUAED tiene entre sus actividades sustantivas el diseñar programas educativos para apoyar la formación y actualización de los docentes; ya sea para quienes transitan o inician sus actividades en las modalidades abierta, a distancia o mixta. Dichos programas están en constante actualización para garantizar una 
Figura 1. Portal del ambiente virtual para el fortalecimiento de la práctica docente (CUAED, 2019b). formación de vanguardia, acorde con las necesidades reales que la práctica docente actual requiere (CUAED, 2019a).

Durante la rectoría del Dr. Enrique Graue, en el marco del Plan de Desarrollo Institucional 2015-2019, a principios de 2017 se inició el diseño de una propuesta de formación docente llamada "Docencia y aprendizaje en la era digital: Trayectorias docentes personalizadas" (ver figura 1). Se trata de una oferta formativa integral, personal y flexible que pretende propiciar la mejora de la calidad del aprendizaje y fortalecer la práctica docente innovadora bajo los principios de equidad, inclusión y atención a la diversidad. Con ello se atiende la necesaria profesionalización y actualización constante del personal académico, al promover el fortalecimiento de su formación mediante cursos en línea orientados a actualizar conocimientos sobre nuevas metodologías de enseñanza, uso apropiado de las TIC y TAC en educación, liderazgo educativo, así como creatividad e innovación en educación. Asimismo, el programa pretende invitar a que los profesores de bachillerato y licenciatura actualicen, de una manera autónoma y flexible, sus conocimientos disciplinarios y didácticos de las asignaturas que atienden, tomando como punto de referencia la capacidad académica desarrollada en cada momento.

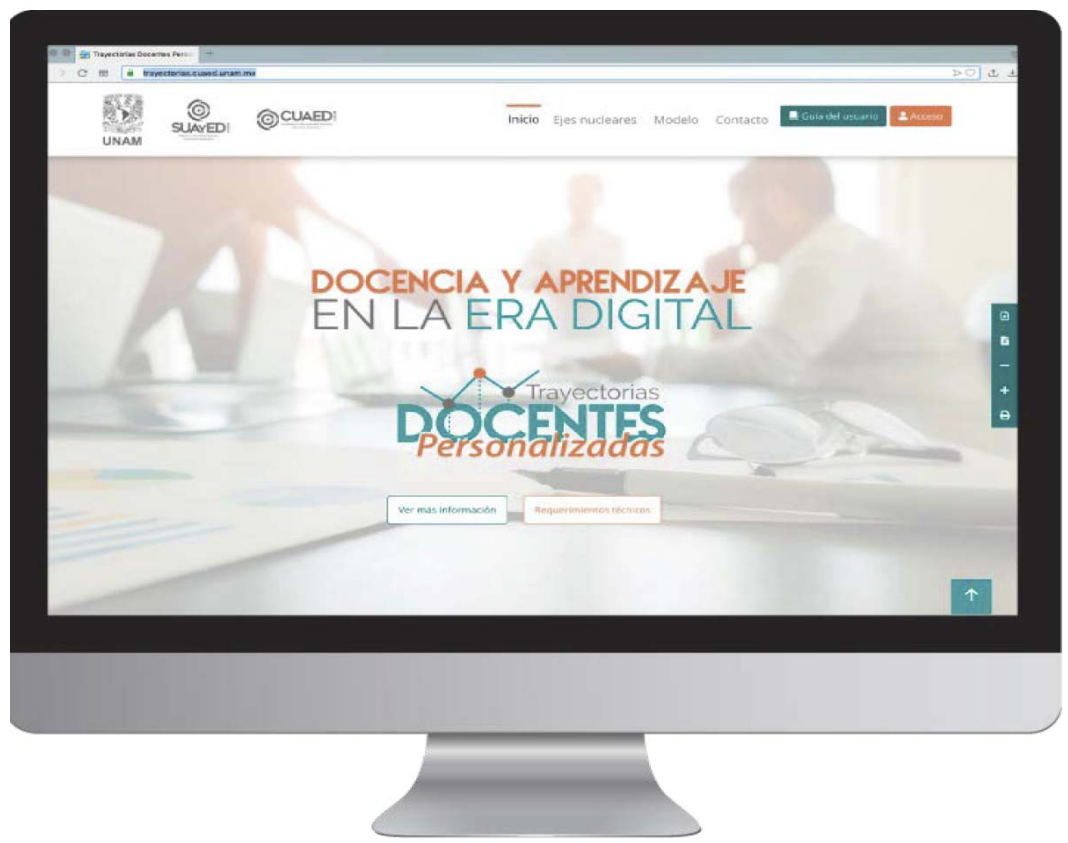

La noción de formación que articula la propuesta alude a un término complejo y multirreferencial al albergar un conjunto de significados que definen al sujeto - a sí mismo- como constructor de sí y, de manera simultánea, en sus relaciones con los otros y en el reconocimiento mutuo, por lo que requiere una lectura desde diferentes ángulos (Ardoino, 1991). Desde la multirreferencialidad, el campo de la formación docente se encuentra configurado en relación con una serie de ciencias y disciplinas que ayudan a explicarla y atenderla, con una diversidad de metodologías y herramientas de indagación. Bajo esta perspectiva, se definieron los ejes formativos o nucleares que permitieran abordar la docencia 
Figura 2. Oferta de cursos en línea del portal Trayectorias docentes personalizadas (CUAED, 2019b) desde diversos campos de conocimiento interrelacionados, a través de temas de frontera y emergentes en el campo educativo y tecnológico.

Asimismo, se recupera la noción de competencia desde una perspectiva holística. El concepto de competencia representa la capacidad de movilizar varios recursos cognitivos para hacer frente a un tipo de situaciones. Las competencias no son en sí mismas conocimientos, habilidades o actitudes; aunque movilizan tales recursos de manera única, es decir, en contextos específicos (Perronoud, 2007). Las competencias profesionales del docente se desarrollan en un proceso de formación y en escenarios de trabajo, en los que juegan un importante papel el conocimiento teórico-práctico y la actividad reflexiva sobre la práctica (Barrón, 2009).

Con base en lo anterior, el modelo curricular del programa se compone de nueve ejes formativos: tecnológico, epistemológico, filosófico, pedagógico, didáctico, psicológico, de la comunicación, socio-histórico-económico y de gestión. En cada eje se consideraron cuatro niveles de dominio de la competencia: a) receptivo, alusivo a la recepción y comprensión de la información; b) resolutivo, concerniente a la resolución de problemas sencillos; c) autónomo, relativo a la actuación con criterio propio y argumentación, y d) estratégico, referente a la aplicación creativa de estrategias (ver figura 2).

\begin{tabular}{|c|c|c|c|c|c|c|c|c|c|}
\hline \multirow[b]{2}{*}{$\begin{array}{c}\text { Niveles de } \\
\text { dominio de las } \\
\text { competencias }\end{array}$} & \multicolumn{9}{|c|}{ Ejes nucleares } \\
\hline & $\begin{array}{c}\text { Tecnológico } \\
\text { \%\% }\end{array}$ & $\begin{array}{c}\text { Psicológico } \\
\text { \&. }\end{array}$ & Pedagógico & $\begin{array}{l}\text { Didáctico } \\
\text { क्ष. }\end{array}$ & $\begin{array}{c}\text { Sociohistórico- } \\
\text { económico } \\
\text { (1) }\end{array}$ & $\begin{array}{c}\text { Filosófico } \\
\text { Ф }\end{array}$ & $\begin{array}{c}\text { Epistemológico } \\
\Sigma\end{array}$ & $\begin{array}{c}\text { De la } \\
\text { comunicación }\end{array}$ & De gestión \\
\hline Receptivo & $\begin{array}{c}\text { Habilidades } \\
\text { digitales }\end{array}$ & $\begin{array}{l}\text { Aprendizaje } \\
\text { centrado } \\
\text { en el } \\
\text { estudiante }\end{array}$ & $\begin{array}{l}\text { Introducción } \\
\text { a las } \\
\text { pedagogías } \\
\text { emergentes }\end{array}$ & $\begin{array}{c}\text { Currículum y } \\
\text { práctica } \\
\text { docente }\end{array}$ & $\begin{array}{c}\text { Globalización } \\
y \\
\text { educación }\end{array}$ & $\begin{array}{l}\text { Introducción } \\
\text { a la teoría } \\
\text { de la } \\
\text { argumentación }\end{array}$ & $\begin{array}{c}\text { Epistemología } \\
\text { de la } \\
\text { educación }\end{array}$ & $\begin{array}{c}\text { Didáctica y } \\
\text { comunicación } \\
\text { digital }\end{array}$ & $\begin{array}{c}\text { Gestión } \\
\text { escolar y de los } \\
\text { aprendizajes } \\
\text { en entornos } \\
\text { virtuales y } \\
\text { presenciales }\end{array}$ \\
\hline Resolutivo & $\begin{array}{l}\text { Ética en } \\
\text { la web }\end{array}$ & $\begin{array}{c}\text { Estilos de } \\
\text { enseñanza } \\
\text { en entornos } \\
\text { virtuales }\end{array}$ & $\begin{array}{c}\text { Intervenciones } \\
\text { didácticas con } \\
\text { pedagogías } \\
\text { emergentes }\end{array}$ & $\begin{array}{l}\text { De los } \\
\text { programas } \\
\text { a las } \\
\text { secuencias } \\
\text { didácticas }\end{array}$ & $\begin{array}{l}\text { Competencias } \\
\text { laborales y } \\
\text { profesionales } \\
\text { en el siglo XXI }\end{array}$ & $\begin{array}{c}\text { Género, } \\
\text { violencia y } \\
\text { cuidado de sí }\end{array}$ & $\begin{array}{c}\text { Conocimiento } \\
\text { y práctica } \\
\text { docente }\end{array}$ & $\begin{array}{c}\text { Comunicación } \\
\text { asertiva en } \\
\text { entornos } \\
\text { digitales }\end{array}$ & $\begin{array}{l}\text { Gestión del } \\
\text { currículum }\end{array}$ \\
\hline \multirow{2}{*}{ Autónomo } & $\begin{array}{c}\text { Recursos } \\
\text { digitales con } \\
\text { fines } \\
\text { didácticos }\end{array}$ & \multirow{2}{*}{$\begin{array}{l}\text { Procesos } \\
\text { para el } \\
\text { aprendizaje } \\
\text { autónomo }\end{array}$} & \multirow{2}{*}{$\begin{array}{l}\text { Implementación } \\
\text { de pedagogías } \\
\text { emergentes }\end{array}$} & \multirow{2}{*}{$\begin{array}{l}\text { Métodos } \\
\text { innovadores } \\
\text { para fomentar } \\
\text { el aprendizaje } \\
\text { autogestivo y } \\
\text { colaborativo }\end{array}$} & \multirow{2}{*}{$\begin{array}{l}\text { Tendencias } \\
\text { de inversión } \\
\text { en capital } \\
\text { humano }\end{array}$} & \multirow{2}{*}{$\begin{array}{l}\text { Educación: } \\
\text { eticidad } \\
\text { y ciudadanía }\end{array}$} & \multirow{2}{*}{$\begin{array}{c}\text { Modelos } \\
\text { pedagógicos } \\
\text { y sus } \\
\text { implicaciones } \\
\text { prácticas en } \\
\text { la docencia }\end{array}$} & \multirow{2}{*}{$\begin{array}{c}\text { Estrategias de } \\
\text { comunicación } \\
\text { para la } \\
\text { educación en } \\
\text { línea }\end{array}$} & \multirow{2}{*}{$\begin{array}{l}\text { Gestión para la } \\
\text { evaluación y } \\
\text { seguimiento } \\
\text { de los } \\
\text { aprendizajes } \\
\text { y la evaluación } \\
\text { institucional }\end{array}$} \\
\hline & $\begin{array}{l}\text { Estrategias } \\
\text { didácticas } \\
\text { apoyadas } \\
\text { por las TIC }\end{array}$ & & & & & & & & \\
\hline Estratégico & $\begin{array}{c}\begin{array}{c}\text { Creación de } \\
\text { espacios de } \\
\text { colaboración }\end{array} \\
\text { Diseño de } \\
\text { entornos } \\
\text { virtuales de } \\
\text { aprendizaje }\end{array}$ & $\begin{array}{l}\text { Docencia y } \\
\text { procesos } \\
\text { afectivos }\end{array}$ & $\begin{array}{l}\text { Análisis de las } \\
\text { propuestas } \\
\text { pedagógicas } \\
\text { emergentes }\end{array}$ & $\begin{array}{c}\text { Evaluación } \\
\text { del aprendizaje } \\
\text { en ambientes } \\
\text { virtuales }\end{array}$ & $\begin{array}{l}\text { Docencia y } \\
\text { globalización }\end{array}$ & $\begin{array}{c}\text { Modelos } \\
\text { teóricos para } \\
\text { el análisis } \\
\text { de la } \\
\text { argumentación }\end{array}$ & $\begin{array}{l}\text { Complejidad y } \\
\text { conocimiento } \\
\text { en la era digital }\end{array}$ & $\begin{array}{l}\text { La } \\
\text { comunicación } \\
\text { en modelos } \\
\text { pedagógicos } \\
\text { emergentes }\end{array}$ & $\begin{array}{l}\text { Diseño y } \\
\text { gestión } \\
\text { de proyectos } \\
\text { educativos }\end{array}$ \\
\hline
\end{tabular}


"Trayectorias personalizadas docentes: fortalecimiento flexible en la práctica docente"

El modelo formativo que subyace a la propuesta recupera la figura docente como un sujeto que aprende, por ello se puntualizan los siguientes aspectos:

a) Centrado en el aprendizaje (learner centredness);

b) Aprendizaje situado, ofrece situaciones reales y problemas a resolver como se presentan en la práctica docente;

c) Pertinencia, vincula los desafíos y problemáticas con las actividades cotidianas de los docentes;

d) Inclusivo e incluyente, diseñado para incorporar a personas con discapacidad auditiva, visual o física;

e) Asesoría, orienta al participante a través de la "Guía del usuario" y de una "Mesa de ayuda", asegurando el acompañamiento académico a lo largo del proceso educativo;

f) Innovación educativa, establece métodos y contenidos innovadores apoyados en tecnologías de vanguardia para la construcción de los entornos virtuales para el aprendizaje;

g) Valores, que promueven la inclusión, la equidad y la justicia social;

h) Usabilidad, navegación sencilla y clara a través de la plataforma Moodle;

i) Accesibilidad: permite personalizar el curso en función de las necesidades específicas del usuario, como disminución del tamaño de la tipografía, poner los textos en alto o bajo contraste, así como habilitar lectores de pantalla;

j) Diseño responsivo, posibilidad de realizar actividades desde cualquier dispositivo (móvil, iPad, o computadora), y

k) Confidencialidad y privacidad, del uso y manejo de datos personales.

Cabe destacar que el modelo se orienta a partir de cuatro principios rectores: a) autoorganización, referido a la capacidad de autorregulación, autoproducción e identidad del participante, b) autorreferencia, búsqueda del autoconocimiento a través de un proceso reflexivo, para propiciar que el aprendiz regule sus emociones; c) autogestión, obtención de conocimientos e información por cuenta propia y a su ritmo, con miras a construir estrategias que favorezcan la autonomía; y, d) dialógico, posibilidad de interactuar en diversos contextos, ambientes, narrativas y lenguajes, conocimientos imprescindibles para la vida.

Cada eje formativo cuenta con las competencias generales esperadas de los docentes al finalizar cada uno de los 38 cursos en línea que componen la oferta; los ejes se trabajan de manera autogestiva. Los cursos se ubican en un nivel de dominio de la competencia a desarrollar, a través de desafíos y actividades. Para su planeación didáctica, se consideró que cada situación de aprendizaje estuviera inscrita en una actividad precedente y en la organización de una actividad subsecuente, con la intención de propiciar la integración de los aprendizajes (ver figura 3). 
Figura 3. Estructura del PEAD (CUAED, 2019b).

\section{ESTRUCTURA DEL PROGRAMA}

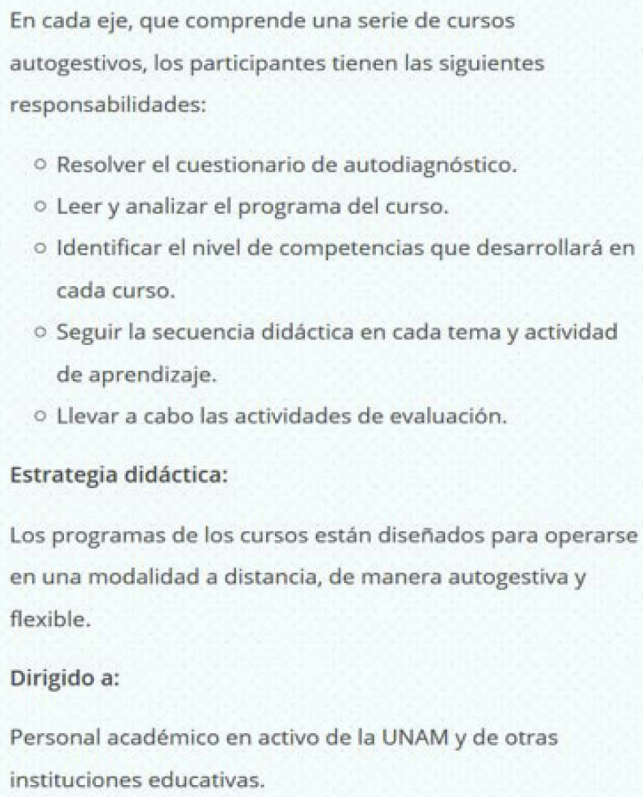

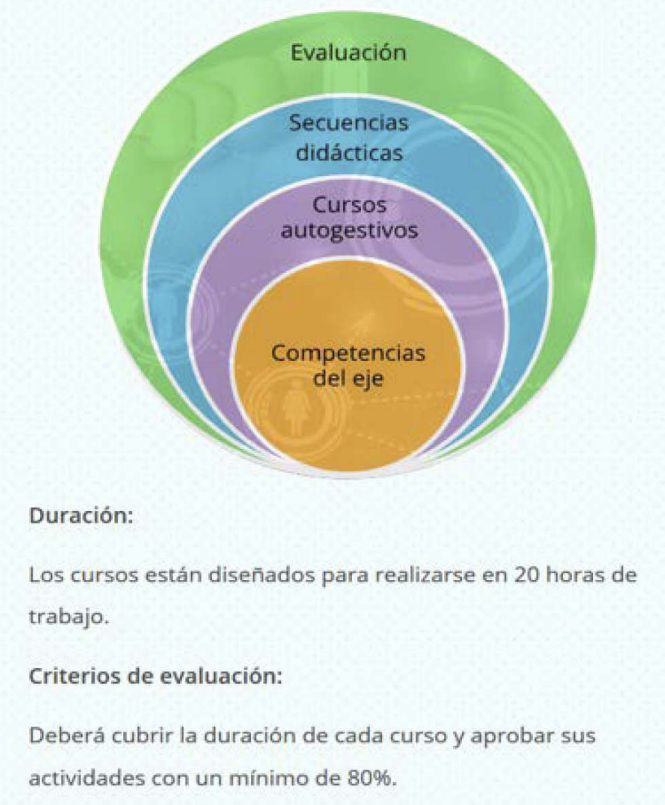

Con base en las competencias a desarrollar se elaboró un instrumento diagnóstico por eje, con el propósito de ubicar al participante en el nivel de dominio de la competencia (receptivo, resolutivo, autónomo o estratégico) y sugerirle el curso de inicio correspondiente. Es obligatorio comenzar por el eje tecnológico, ya que se requieren desarrollar habilidades digitales básicas para trabajar en los otros ejes; al concluirlo el participante podrá elegir libremente el eje de su interés y/o necesidad, construyendo así su trayectoria personalizada. A partir del autodiagnóstico, el docente tendrá la posibilidad de ubicar aquellas competencias que desea integrar a su esquema cognitivo, en función de sus necesidades, intereses y actividades.

La evaluación del aprendizaje es formativa, se lleva a cabo de manera permanente para detectar dificultades, comprenderlas y autorregularlas, cabe señalar que para la aprobación de los cursos se tendrán que realizar todas las actividades propuestas. Asimismo, el docente estará en posibilidad de guardar los productos elaborados en los cursos en un portafolio personal, que podrá consultar en cualquier momento.

La dedicación requerida por el participante es de 20 horas por curso. Se tendrán que aprobar las actividades con un mínimo de $80 \%$ de aciertos para la obtención de su constancia digital, la cual se generará de manera automática, una vez concluido el módulo. Finalmente, también se elaboró la Guía del usuario para la comprensión del modelo operativo considerado en el diseño de la plataforma, que se desarrolló utilizando el LMS MOODLE, versión 3.1.7. 
"Trayectorias personalizadas docentes: fortalecimiento flexible en la práctica docente" María Concepción Barrón Tirado y Francisco Cervantes Pérez Vol. 20, Núm. 6, noviembre-diciembre 2019 Revista Digital Universitaria

\section{Conclusiones}

Debido a la diversidad de perfiles en los docentes considerados como población objetivo de este proyecto y a la cantidad de información que se manejará en la plataforma tecnológica, se ha requerido la coordinación general de un equipo multidisciplinario formado por un coordinador académico, coordinadores y académicos expertos en los contenidos ${ }^{1}$ de cada uno de los ejes temáticos, diseñadores pedagógicos, integradores web, correctores de estilo, programadores e ingenieros.

Durante la primera etapa, en el verano de 2018, se invitó a profesores del SUAYED a participar en una prueba piloto para evaluar el sistema y el contenido de los 38 cursos, respondiendo al Ilamado un total de 40 académicos. Asimismo, de octubre de 2018 a febrero de 2019, el eje tecnológico fue piloteado, en formato de diplomado, por un grupo de 116 profesores de la Maestría en Educación Media Superior (MADEMS), quienes contaron con el apoyo en línea de tres académicos de la CUAED que fungieron como monitores-asesores. Los resultados obtenidos en ambos procesos permitieron reestructurar todos los cursos y se hicieron las adecuaciones necesarias en el sistema. De manera paralela, se solicitó a diversos especialistas su opinión en torno al modelo propuesto y los contenidos planteados. Una vez recuperadas las opiniones y tras realizar las adecuaciones pertinentes, se procedió a su difusión ante la comunidad universitaria. A partir del 19 de marzo de 2019, se liberó el sitio público, y para el mes de julio del mismo año se contaba ya con alrededor de mil académicos-docentes de la UNAM fortaleciendo su práctica docente en la plataforma.

¿Hacia dónde vamos? El programa será evaluado de manera permanente a través de diversos cuestionarios incorporados en los cursos, con el fin de dar seguimiento al proyecto y actualizar lo que se vaya requiriendo. De esta forma, el programa "Docencia y aprendizaje en la era digital: Trayectorias docentes personalizadas" se erige como una propuesta al servicio de la comunidad universitaria con miras a mejorar sus prácticas en distintos ambientes educativos, misma que la Dirección General de Asuntos de Personal Académico (DGAPA) ha validado y otorgado su reconocimiento como una opción en línea para la superación del personal académico de nuestra máxima casa de estudios.

La construcción de trayectorias docentes personalizadas constituye un salto cualitativo al considerar la participación del docente en función de sus intereses, características, necesidades y posibilidades de fortalecimiento en su formación. Asimismo, representa la oportunidad de trazar una ruta a seguir de manera

${ }^{1}$ Académicos tanto de la UnAm como diversas instituciones educativas del país, quienes cuentan con amplia experiencia en distintos niveles de educación y en diversas modalidades, ya sea presencial, abierta o a distancia. personal, que no se limite a lugares, situaciones y contextos institucionales, abriéndose opciones para incursionar en nuevos nichos de aprendizaje que se pueden cultivar a lo largo de la vida. 


\section{Referencias}

* Ardoino,J. (1991). El análisis multirreferencial. Recuperado de: http://publicaciones. anuies.mx/pdfs/revista/Revista87 S1A1ES.pdf.

* Barrón Tirado, C. (2009). Docencia universitaria y competencias didácticas. Perfiles Educativos, XxxI (125), pp. 76-87.

- Coll, C. (2013). El currículo escolar en el marco de la nueva ecología del aprendizaje. Aula de Innovación Educativa, 210, 31-36.

- Coll, C. (2016). La personalización del aprendizaje escolar, una exigencia de la nueva ecología del aprendizaje. España: EDuforics. Recuperado de: http://www.eduforics. com/es/la-personalizacion-del-aprendizaje-escolar-una-exigencia-de-la-nuevaecologia-del-aprendizaje/.

* Coordinación de Universidad Abierta y Educación a Distancia (CUAED) (2019a). Acerca de la cuaed. México: unam. Recuperado de: https://web.cuaed.unam.mx/ acerca-de-la-cuaed/.

* Coordinación de Universidad Abierta y Educación a Distancia (CUAED), (2019b). Trayectorias docentes personalizadas. México: unam. Recuperado de: https:// trayectorias.cuaed.unam.mx/.

* Graue, E. (2017). Plan de Desarrollo Institucional 2015-2019. México: unam.

* Perrenoud, P. (2007). Diez nuevas competencias para enseñar. México: Editorial Grao.

\section{Cómo citar este artículo}

* Barrón Tirado, María Concepción y Cervantes Pérez, Francisco (2019). Trayectorias personalizadas docentes: fortalecimiento flexible en la práctica docente. Revista Digital Universitaria (RDU). Vol. 20, núm. 6 noviembre-diciembre. Dol: http://doi. org/10.22201/codeic.16076079e.2019.v20n6.a3.

Recepción: 23/07/2019. Aprobación: 19/09/2019 